\title{
Competing risks need to be considered in survival analysis models for cardiovascular outcomes
} \author{
Martin Schumacher, Dr rer Nat ${ }^{b}$

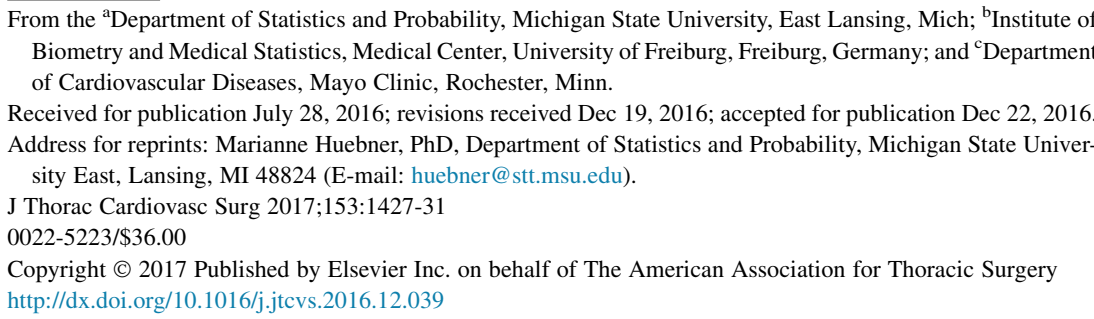

Marianne Huebner, PhD, ${ }^{\mathrm{a}}$ Martin Wolkewitz, Dr Sc Hum, ${ }^{\mathrm{b}}$ Maurice Enriquez-Sarano, MD, ${ }^{\mathrm{c}}$ and
Benefits of interventions for patients with cardiovascular diseases are often studied considering the time since the intervention to the occurrence of an outcome of interest. The Kaplan-Meier estimator is commonly used to calculate survival functions and takes into account the number of occurrences of a primary outcome and time to occurrence, whereas Cox proportional hazard models include covariates with potential impact on survival. ${ }^{1}$ An important consideration is censoring, namely when the event of interest is not observed due to end of study, loss to follow-up, or competing events that preclude occurrence of the outcome. For example, congestive heart failure (CHF) after mitral valve repair considers time from the surgery to heart failure. The patient may die before a diagnosis of a CHF could have been made, and thus the censoring assumption that censored observations have the same CHF hazard as those at-risk is not fulfilled, so competing outcomes may need to be considered. Another example is regarding the age of the patient. Patients develop cardiovascular disease at different ages. Because older patients are at high risk for death before experiencing a CHF, it might appear that older age could be "protective" for the cumulative CHF incidence. Competing risk models allow for flexible modeling, provide valid estimates for cumulative incidences, and might explain apparently counterintuitive findings. This was also examined for patients with coronary bypass surgery and re-interventions, ${ }^{2}$ or for patients with implanted cardioverter-defibrillators. ${ }^{3}$ In such situations, the assumption that censored subjects have the same risk as subjects remaining in the cohort is not correct for Kaplan-Meier analysis or Cox models. It has been estimated that $46 \%$ of studies in high-impact medical journals are susceptible to competing risk bias. ${ }^{4}$ Kaplan-Meier estimates and competing risk estimates answer different questions, as has been discussed in the example of heart valve replacement. ${ }^{5}$ The perspectives can be in terms of mortality or valve deterioration, whichever occurs first, or in the hypothetical scenario of valve deterioration without considering

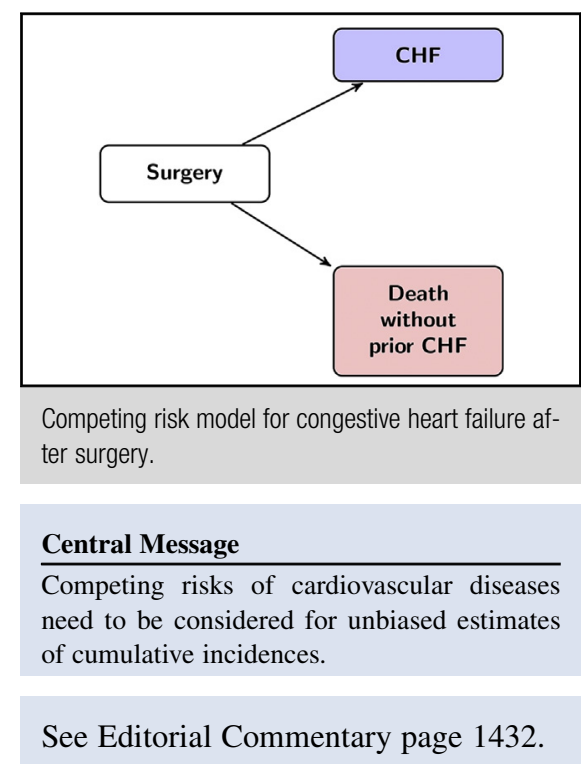

mortality. The problems and opportunities with statistical models in the presence of competing risk are explained with the following case study.

\section{EXAMPLE}

Data from a published study on mitral regurgitation for 1218 patients who underwent degenerative mitral valve repair 1996 to 2000 at Mayo Clinic Rochester, Minn, USA, are used for illustration. ${ }^{6}$ Of those patients, 221 experienced $\mathrm{CHF}, 318$ died without $\mathrm{CHF}$, and 134 died after CHF. The mean age in the cohort was 64 (standard deviation 13 years); New York Heart Association (NYHA) Classification III or IV was present in 366 patients $(30 \%)$. The median follow-up time was 11.5 years (interquartile range 4.4 years). Echocardiography was performed postoperatively and during the follow-up time.

\section{MODELS TAKING COMPETING RISKS/MULTIPLE ENDPOINTS INTO ACCOUNT}

The question of what is the risk of CHF after mitral valve surgery can be answered with different models. Cox proportional hazard models assume that patients lost to follow-up have noninformative censoring distribution. This means when heart failure is the primary outcome and death is censored, then the occurrence of death has no influence on the probability of CHF. This is unlikely, and the hazard ratios (HRs) from such models cannot be interpreted as a 
comparison of cumulative incidences. An alternative is to consider a composite endpoint, in which time since surgery is calculated to the time of CHF or death, whichever occurs first (Figure 1, A). A common HR for a composite endpoint is efficient, because events for both outcomes are combined to achieve good statistical power. However, it is not possible to show efficacy for individual components of the composite endpoint. Freemantle et $\mathrm{al}^{7}$ discussed further advantages

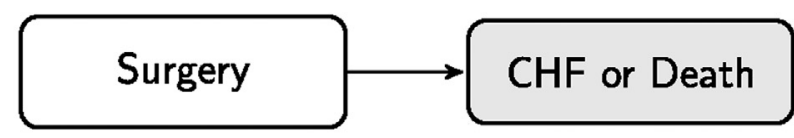

A

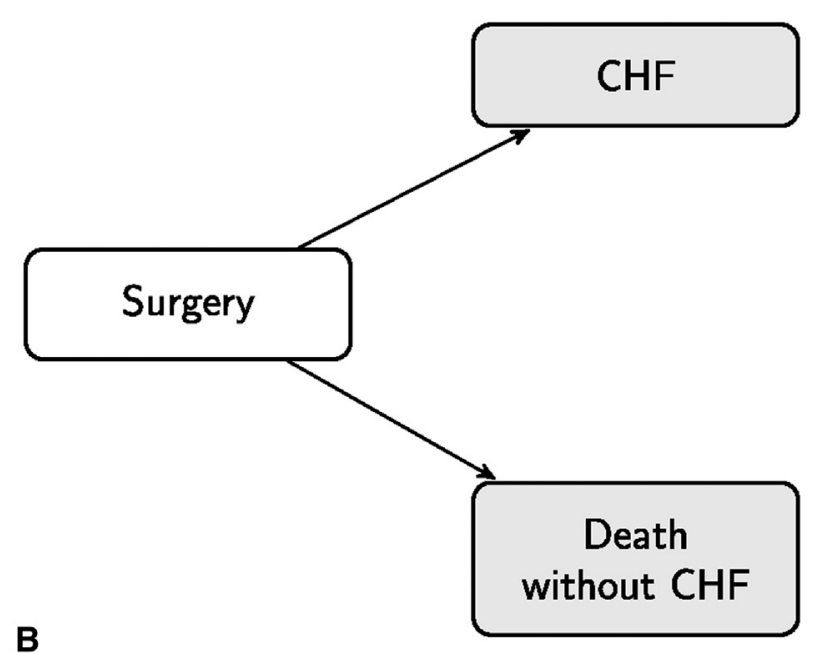

B

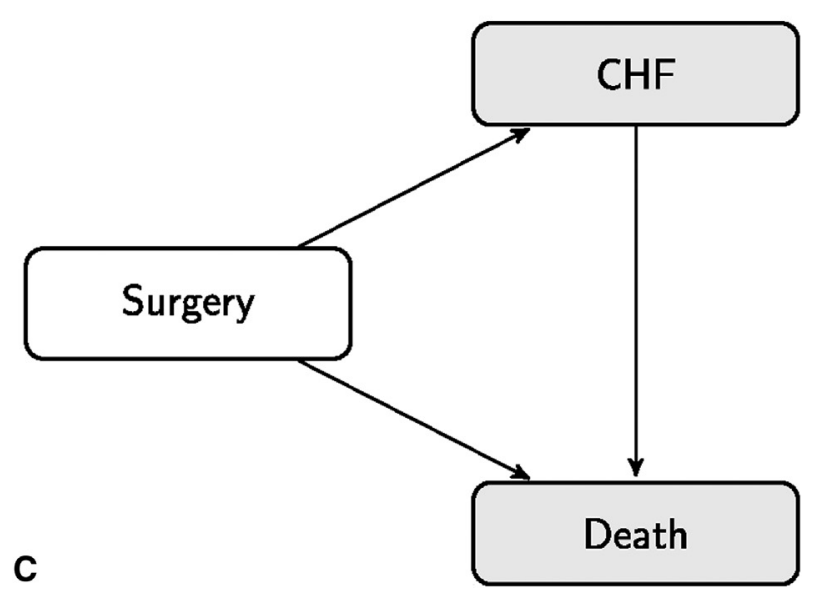

FIGURE 1. Competing risk models extend time-to-event models. A, Model for CHF after surgery. B, Competing risk model for CHF-free survival. C, Multistate model for survival accounting for $\mathrm{CHF}$ as intermediate event. $\mathrm{CHF}$, Congestive heart failure. and disadvantages of models with a composite endpoint and appropriate reporting.

We may be interested in the incidence of CHF during a follow-up period while considering that patients may die from the disease without CHF. This is a competing risk scenario with 2 endpoints, CHF and death without CHF, and we consider the time from surgery to CHF, and the time from surgery to death without CHF (Figure 1, B). Cox proportional hazard models can be used for each of the transitions (arrows in the diagram) to calculate HRs, while taking both transitions into account.

Cumulative incidence functions (CIF) at a specific follow-up time are defined as the probability that an event occurs before that time and are calculated for each time point. If such an event is observed for every patient in the sample, then the cumulative incidence can be calculated as the relative frequency of survival times up to the specified follow-up time. If there are incomplete data or censoring such that a patient, who is censored at some time, is representative for those who have not experienced the event ("independent censoring"), the CIF can be estimated as 1 minus the Kaplan-Meier estimator, the probability of surviving (or not experiencing the event) until the follow-up time. In the presence of competing events, the CIF depends on the hazard rate of the event of interest as well as of the hazard rate of the competing events; it can be estimated with the AalenJohansen estimator. ${ }^{8}$ Then, the CIF is the product of the time-dependent probability of being alive at-risk and the conditional probability of experiencing the event of interest. Analogously, the CIF for death without CHF, the product of the time-dependent probability of being CHF-free at-risk and the conditional probability of dying without CHF.

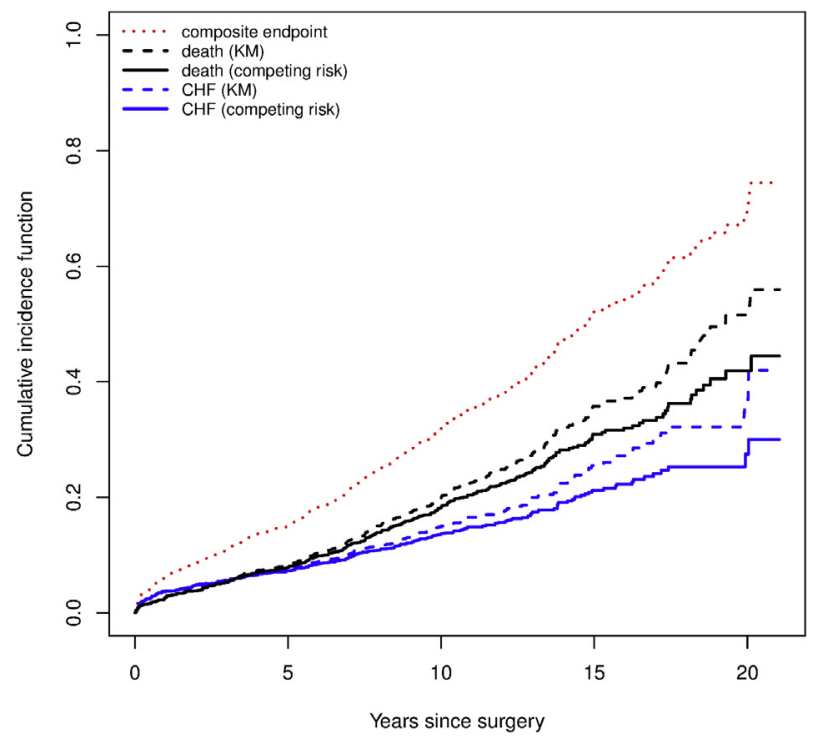

FIGURE 2. Cumulative incidence curves for competing risk models and Kaplan-Meier curves. $C H F$, Congestive heart failure; $K M$, Kaplan-Meier. 
TABLE 1. Cumulative incidence at 15 and 20 years of follow-up in the models with and without competing risks

\begin{tabular}{lcccccc}
\hline Cumulative incidence & Death or CHF & CHF (competing risks) & Death (competing risks) & CHF (KM) & Death (KM) \\
\hline At 15-y follow-up & $52.1 \%(48.2 \%, 55.7 \%)$ & $21.2 \%(18.3 \%, 24.0 \%)$ & $30.9 \%(27.5 \%, 34.1 \%)$ & $25.5 \%(21.8 \%, 29.0 \%)$ & $35.7 \%(31.7 \%, 39.5 \%)$ \\
At 20-y follow-up & $69.3 \%(61.5 \%, 75.6 \%)$ & $27.4 \%(21.8 \%, 32.8 \%)$ & $41.9 \%(35.8 \%, 47.4 \%)$ & $36.7 \%(25.9 \%, 45.9 \%)$ & $51.6 \%(42.9 \%, 58.9 \%)$ \\
\hline CHF, Congestive heart failure; $K M$, Kaplan-Meier.
\end{tabular}

Note that 1 minus the sum of both CIFs yields the timedependent probability of being alive and without CHF. Thus, to calculate the CIF for CHF, the competing event of death also needs to be considered. The CIF can be calculated for composite endpoint or for each, CHF and death. Figure 2 illustrates the estimation of cumulative incidences of the various models for the mitral valve repair data. The classic Kaplan-Meier curves with 1 endpoint and censoring any other events are shown to overestimate the incidences for CHF or death compared with the curves that take competing risk into account. For example, at 20 years, the risk of CHF is $27.5 \%$ when taking competing risks into account and $36.7 \%$ when not. The cumulative incidence at 20 years for the composite endpoint $(69.3 \%)$ is the sum of the incidences for CHF $(27.4 \%)$ and death $(41.9 \%)$ in the competing risk scenario (Table 1).

An extension of competing risk models are multistate models that provide a flexible framework for many situations. The empirical estimator for the transition probabilities in such multistate models is the Aalen-Johansen estimator. ${ }^{8}$ As an example, we consider here estimating not only the mortality risk after surgery, but also the mortality risk after CHF (Figure 1, C).

Age is associated with the risk of the composite endpoint $\mathrm{CHF}$ or death, the HR for 5-year increments is $1.4(95 \%$ confidence interval [CI] 1.3-1.5). The competing risk analysis shows that this age effect is apparent in the effect on death without prior $\mathrm{CHF}, \mathrm{HR}=1.5$ (95\% CI 1.4-1.6); the age effect also exists for CHF, HR $=1.3(95 \%$ CI 1.2 1.4). In contrast to the composite analysis, the HRs cannot be interpreted as a comparison of risks, only as actual HRs. Thus, the older the patient is, the faster he or she dies without CHF or the faster he or she obtains a CHF. In addition, we can estimate the HR for age in the multistate model for the transition death after CHF as 1.5 (95\% CI 1.31.7) (Table 2), meaning that patients with older ages die also faster after CHF. Hence age increases the hazard of all 3 events (CHF, death without CHF, and death after CHF).

The NYHA classification is associated with the composite endpoint CHF or death with an HR of 1.8 (95\% CI 1.52.1). The competing risk model or multistate model shows that a higher NYHA class is also a statistically significant risk factor for CHF, $\mathrm{HR}=1.9$ (95\% CI 1.4-2.5) and for death after $\mathrm{CHF}, \mathrm{HR}=1.6(95 \% \mathrm{CI} 1.1-2.5)$. NYHA class increased the hazard of death without CHF, HR $=1.7(95 \%$ CI 1.3-2.1) (Table 2). This also can be seen in the CIFs for CHF and death without CHF stratified by NYHA class shown in Figure 3.

For the models, the statistical software $\mathrm{R}$ version 3.1.2 (2014-10-31) was used that included the package survival v. 2.38 .1 .

There is a 1-to-1 correspondence between probabilities (Table 1) and rates (Table 2). A higher HR for a factor would mean a higher cumulative incidence. Although the KaplanMeier curves consider only the rate of the event of interest, CIFs for competing events are calculated considering all events simultaneously (Figure 4).

\section{DISCUSSION}

The prevalence and magnitude of competing risk bias has recently been investigated in a random sample of 100 publications in prominent medical journals, ${ }^{4}$ comprising 6 publications from the Journal of Thoracic and Cardiovascular Surgery (JTCVS). Three of those considered overall survival as the primary endpoint, presenting Kaplan-Meier estimates that were adequate for this situation. In the other 3 , however, death was a competing event for the primary endpoints time to reoperation, ${ }^{9}$ time to stable remission, ${ }^{10}$ and time to postoperative extubation. ${ }^{11}$ In all 3 articles, KaplanMeier estimates were presented. Because death as a competing event was not considered, they were judged as susceptible to competing risk bias. ${ }^{4}$ Luckily, the magnitude

TABLE 2. Hazard ratios ( $95 \%$ confidence intervals) for CHF-free survival and for competing risk models

\begin{tabular}{|c|c|c|c|c|}
\hline \multirow{2}{*}{$\frac{\text { Model }}{\text { Outcome }}$} & \multirow{2}{*}{$\begin{array}{c}\text { Survival model } \\
\text { Figure } 1, A \\
\text { CHF or death }\end{array}$} & \multicolumn{2}{|c|}{$\begin{array}{c}\text { Competing risk model } \\
\text { Figure } 1, B \\
\end{array}$} & \multirow{2}{*}{$\frac{\text { Multistate model Figure } 1, C}{\text { Death after CHF }}$} \\
\hline & & $\mathbf{C H F}$ & Death without prior CHF & \\
\hline \multicolumn{5}{|l|}{ Predictors } \\
\hline Age (5-y increments) & $1.4(1.3-1.5)$ & $1.3(1.2-1.4)$ & $1.5(1.4-1.6)$ & $1.5(1.3-1.7)$ \\
\hline NYHA class III or IV vs I or II & $1.8(1.5-2.1)$ & $1.9(1.4-2.5)$ & $1.7(1.3-2.1)$ & $1.6(1.1-2.5)$ \\
\hline
\end{tabular}

Covariates in these models are age (in 5-year increments) and NYHA classification III or IV compared with I or II. All models are adjusted for sex, residual mitral regurgitation, localization, and coronary artery bypass grafting. CHF, Congestive heart failure; NYHA, New York Heart Association. 


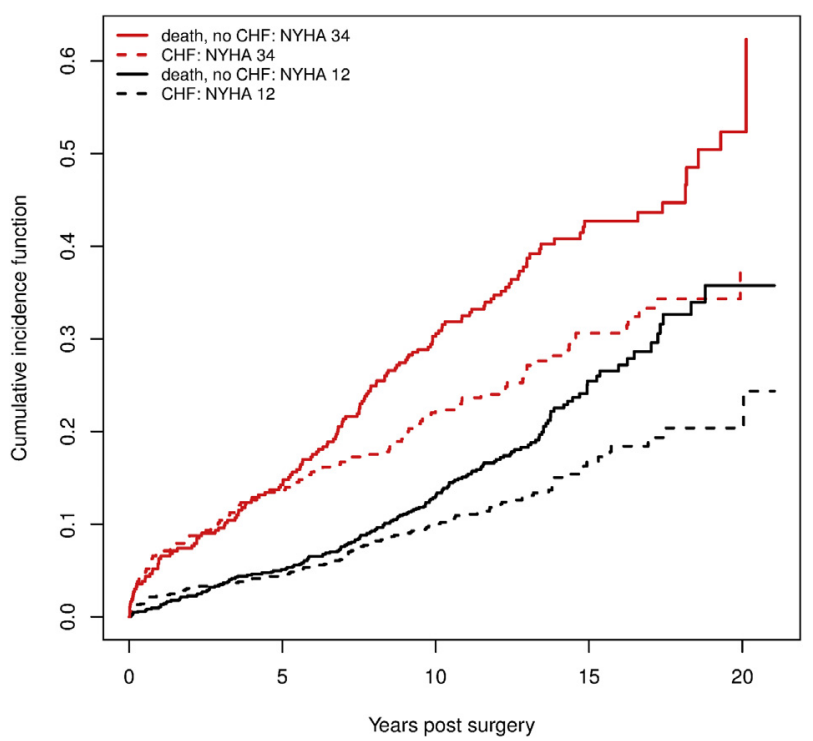

FIGURE 3. Cumulative incidence functions for CHF and death without CHF stratified by NYHA classification. $C H F$, Congestive heart failure; NYHA, New York Heart Association.

of the bias was judged as relatively small in at least 2 of the mentioned publications.

Statistical methodology, such as cumulative incidence curves instead of Kaplan-Meier estimates, may be more adequate when competing risks are present. In the example, the cumulative incidence function estimates the timedependent probability of CHF in a realistic situation that patients could die without CHF within the time period of interest. In contrast, there are attempts to interpret the 1 minus Kaplan-Meier estimate as the probability of CHF in a hypothetical situation that patients will not die without CHF within the period of interest. Thus, predictions based on Kaplan-Meier estimates are often speculative and we do not recommend their usage, especially when communicating risks to patients. For instance, a recent study ${ }^{12}$ provides a Kaplan-Meier plot showing that the probability of surviving the intensive care unit (ICU) is lower than $20 \%$ within 3 months for patients who were admitted during office hours; however, the table showed that this prediction is based on data of patients of whom actually $96 \%$ survived the ICU. CIFs would have provided a more realistic figure by accounting for ICU discharge as a competing event for ICU death.

All model assumptions need to be carefully checked. We did not discuss the Markov property for multistate models. This means that future events only depend on the present state, but extensions of these models under different assumptions are possible.

As with other statistical models, the number of events (not the sample size) limits how many transitions can be estimated without overfitting. Some care needs to be taken

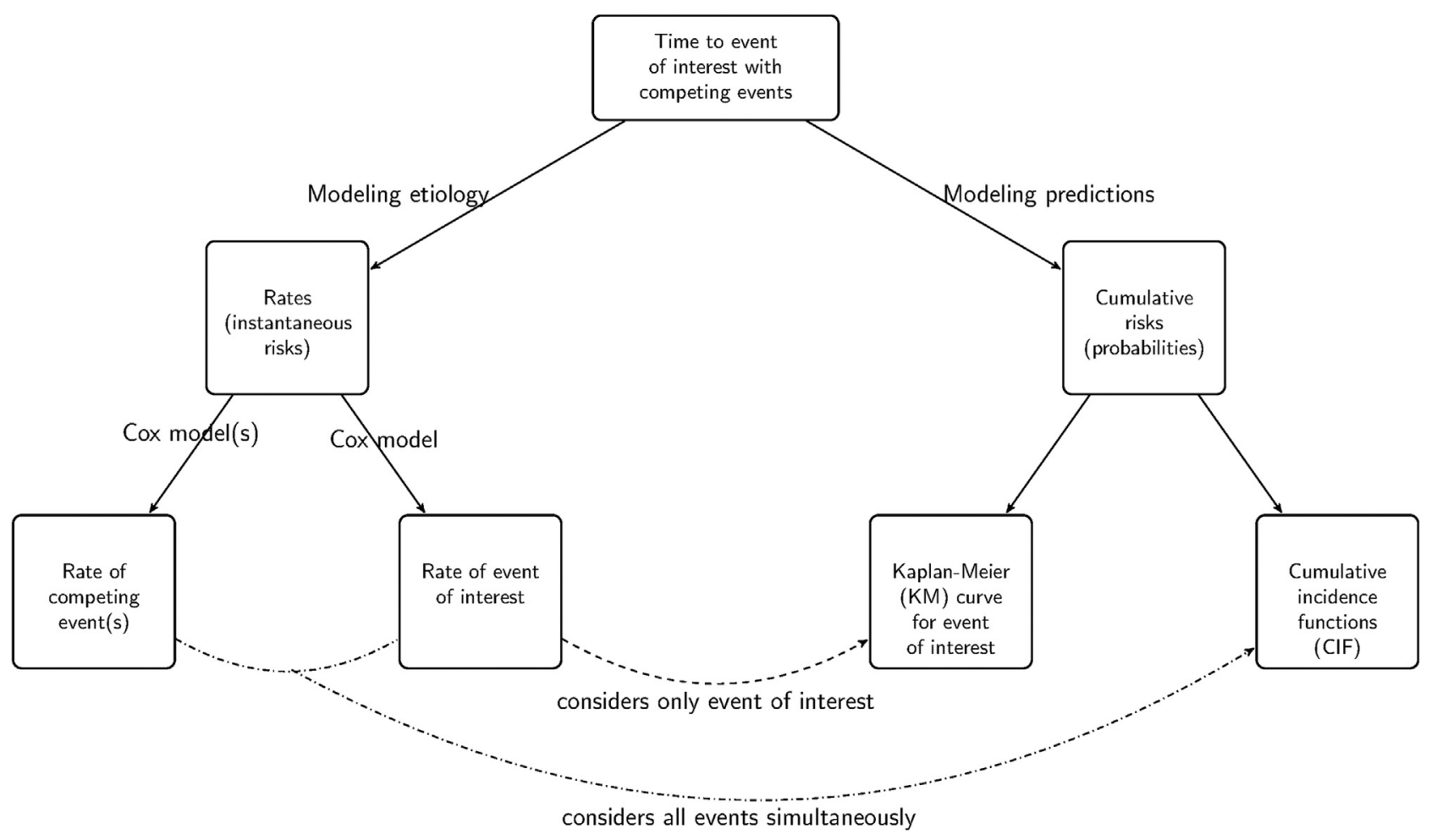

FIGURE 4. Diagram for modeling rates or cumulative incidences in a situation with competing risks. 
when preparing the dataset for analysis when competing events happen on the same day. This is referred to as "ties."

Other multistate models are possible, but are not discussed here. For example, we discussed a model for time to first $\mathrm{CHF}$, but sequential events also are possible in the multistate model framework. Furthermore, competing risk models also can account for several competing events, such as death from different causes, cardiovascular death, cancer, or other causes. $^{13-17}$ There are several multivariable modeling approaches, based on cause-specific or on subdistribution hazards. The choice of these models needs careful consideration of the research question. ${ }^{17}$

\section{CONCLUSIONS}

The presence of competing events to a primary endpoint will lead to biased risk estimates when the competing events are censored. CIFs for the primary endpoint as well as the competing events should be used instead. Competing risk models allow for analysis of multiple endpoints. There are rich possibilities of modeling cardiovascular diseases with competing risk or multistate models when considering transitions between multiple states or sequential events.

\section{Conflict of Interest Statement}

Authors have nothing to disclose with regard to commercial support.

\section{References}

1. Therneau TM, Grambsch PM. Modeling Survival Data: Extending the Cox Model. New York: Springer; 2001.

2. Blackstone EH, Lytle BW. Competing risks after coronary bypass surgery: the influence of death on reintervention. J Thorac Cardiovasc Surg. 2000;119: 1221-30.
3. Wolbers M, Koller MT, Stel VS, Schaer B, Jager KJ, Leffondre K, et al. Competing risks analyses: objectives and approaches. Eur Heart J. 2014;35: 2936-41.

4. van Walraven C, McAlister FA. Competing risk bias was common in Kaplan Meier risk estimates published in prominent medical journals. J Clin Epidemiol. 2016;69:170-3.e8.

5. Bodnar E, Blackstone EH. Editorial: an "actual" problem: another issue of apples and oranges. J Heart Valve Dis. 2005;14:706-8.

6. Suri RM, Clavel M-A, Schaff HV, Michelena HI, Huebner M, Nishimura RA, et al. Effect of recurrent mitral regurgitation following degenerative mitral valve repair: long-term analysis of competing outcomes. J Am Coll Cardiol. 2016;67: 488-98.

7. Freemantle N, Calvert M, Wood J, Eastaugh J, Griffin C. Composite outcomes in randomized trials: greater precision but with greater uncertainty? JAMA. 2003 289:2554-9.

8. Aalen OO, Johansen S. An empirical transition matrix for nonhomogeneous Markov chains based on censored observations. Scand J Stat. 1978;5:141-50.

9. Kari FA, Liang DH, Kvitting J-P, Stephens EH, Mitchell RS, Fischbein MP, et al. Tirone David valve-sparing aortic root replacement and cusp repair for bicuspid aortic valve disease. J Thorac Cardiovasc Surg. 2013;145:35-40.e1-2.

10. Mineo TC, Ambrogi V. Outcomes after thymectomy in class I myasthenia gravis. J Thorac Cardiovasc Surg. 2013;145:1319-24.

11. Redlin M, Kukucka M, Boettcher W, Schoenfeld H, Huebler M, Kuppe H, et al Blood transfusion determines postoperative morbidity in pediatric cardiac surgery applying a comprehensive blood-sparing approach. J Thorac Cardiovasc Surg. 2013;146:537-42.

12. Ju M-J, Tu G-W, Han Y, He HY, He YZ, Mao HL, et al. Effect of admission time on mortality in an intensive care unit in Mainland China: a propensity score matching analysis. Crit Care. 2013;17:R230.

13. Kouchoukos NT, Blackstone EH, Hanley FL, Kirklin JK. Kirklin/BarrattBoyes Cardiac Surgery: Expert Consult-Online and Print. 4th ed. Philadelphia, PA: Saunders; 2012.

14. Haller B, Schmidt G, Ulm K. Applying competing risks regression models: an overview. Lifetime Data Anal. 2013;19:33-58.

15. Putter H, Fiocco M, Geskus RB. Tutorial in biostatistics: competing risks and multi-state models. Stat Med. 2007;26:2389-430.

16. Andersen PK, Geskus RB, de Witte T, Putter H. Competing risks in epidemiology: possibilities and pitfalls. Int J Epidemiol. 2012;41:861-70.

17. Austin PC, Lee DS, Fine JP. Introduction to the analysis of survival data in the presence of competing risks. Circulation. 2016;133:601-9. 